\title{
Convergence Properties for Uncertain Sequence
}

\author{
Cuilian You and Lijuan Yan \\ College of Mathematics and Information Science, Hebei University, Baoding 071002, China \\ Correspondence should be addressed to Cuilian You; yycclian@163.com
}

Received 13 July 2017; Accepted 8 October 2017; Published 1 November 2017

Academic Editor: Francesco Marotti de Sciarra

Copyright (C) 2017 Cuilian You and Lijuan Yan. This is an open access article distributed under the Creative Commons Attribution License, which permits unrestricted use, distribution, and reproduction in any medium, provided the original work is properly cited.

Uncertainty theory is a branch of axiomatic mathematics for modeling belief degrees. Since convergence for uncertain sequences plays a very important role in uncertainty theory and applications, we investigated some properties of convergence in measure in this paper. In addition, some theorems and properties of convergence in $p$-distance were studied.

\section{Introduction}

Uncertainty theory which is founded by Liu [1] has been used to model uncertain phenomena for a long time. During the past years, many researchers contributed a lot to this area. For instance, Liu [2] introduced a classical application of uncertainty theory. In order to indicate the belief degree that an event happens, Liu [1] defined a definition of uncertain measure as a set function satisfying normality, duality, subadditivity, and product axiom. Liu [1] put forward the concept of uncertain variable. Then Liu and $\mathrm{Ha}$ [3] presented a formula to calculate the expected value of a function of uncertain variables. After that, Liu [4] introduced uncertain programming, and Li and Liu [5] studied uncertain logic. Liu [6] founded uncertain process which is essentially a sequence of uncertain variables indexed by time, and then the uncertain distribution and independence of uncertain process were studied by Liu [7] in 2014. Furthermore, uncertain differential equation was also given by Liu [6] and further researched by Chen and Liu [8] whose main content is existence and uniqueness theorem for uncertain differential equation. To date, uncertainty theory has been already applied to uncertain risk measure (Liu [2]), uncertain set (Liu [9]), uncertain inference (Gao et al. [10]), and so on.

Since sequence convergence is a useful tool in the fundamental theory of mathematics, some definitions of sequences convergence of uncertain sequences were proposed by Liu [1] and You [11]. The Cauchy convergence of uncertain sequences and the sufficient conditions of convergence almost surely were presented by Xia [12]. H. Wang and Z. Wang [13] studied the dual limits of uncertain variables sequence in 2013. Then, Gao [14] discussed the convergence theorems for expected value of uncertain variables. A necessary and sufficient condition of convergence in measure for uncertain sequence was presented by Guo et al. [15]. Therefore, $r$-order convergence and dual convergence of uncertain variable sequences were researched by Yuan [16] and Yuan et al. [17]. Chen et al. [18] introduced the convergence concepts of complex uncertain sequence. In addition, You and Yan [19] gave a new concept of sequence convergence for uncertain variables that is convergence in $p$-distance; then the relationships among concepts of convergence for uncertain variables sequences were studied by You and Yan [20] in 2016.

The main objective of this paper is to research the properties of convergence for uncertain sequence. The structure of the paper is as follows: we shall briefly introduce uncertain space and some basic contents and theorems of uncertainty theory in Section 2. Then some new properties of convergence in measure and convergence in $p$-distance will be investigated in Section 3. Finally, a brief summary will be given.

\section{Preliminary}

Some definitions and theorems of uncertainty theory are presented in this section.

Let $\Gamma$ be a nonempty set, and let $\mathscr{L}$ be a $\sigma$-algebra over $\Gamma$. Each element $\Lambda$ in $\mathscr{L}$ is called an event. To describe the belief 
degree that each event will happen, Liu [1] proposed the set function $\mathscr{M}$, which is called uncertain measures if it satisfies the following three axioms.

Axiom 1 (normality). $\mathscr{M}\{\Gamma\}=1$.

Axiom 2 (self-duality). $\mathscr{M}\{\Lambda\}+\mathscr{M}\left\{\Lambda^{c}\right\}=1$, for any event $\Lambda$.

Axiom 3 (subadditivity). For every countable sequence of events $\left\{\Lambda_{i}\right\}$, we have

$$
\mathscr{M}\left\{\bigcup_{i=1}^{\infty} \Lambda_{i}\right\} \leq \sum_{i=1}^{\infty} \mathscr{M}\left\{\Lambda_{i}\right\}
$$

Next, we will introduce the definition of uncertainty space.

Definition 1 (see Liu [1]). Let $\Gamma$ be a nonempty set, let $\mathscr{L}$ be a $\sigma$-algebra over $\Gamma$, and let $\mathscr{M}$ be an uncertain measure. Then the triplet $(\Gamma, \mathscr{L}, \mathscr{M})$ is called an uncertainty space.

For uncertain measure, we have the following theorem.

Theorem 2 (monotonicity, Liu [1]). Uncertain measure $\mathscr{M}$ is a monotone increasing set function. That is, for any events $\Lambda_{1} \subset$ $\Lambda_{2}$, we have $\mathscr{M}\left\{\Lambda_{1}\right\} \leq \mathscr{M}\left\{\Lambda_{2}\right\}$.

Definition 3 (see Liu [1]). An uncertain variable is a function $\xi$ from an uncertainty space $(\Gamma, \mathscr{L}, \mathscr{M})$ to the set of real numbers such that $\{\xi \in B\}$ is an event for any set $B$ of real numbers.

Then some convergence concepts and theorems of uncertain sequence were given.

Definition 4 (see Liu [1]). The uncertain sequence $\left\{\xi_{i}\right\}$ is said to be convergent in measure to $\xi$ if

$$
\lim _{i \rightarrow \infty} \mathscr{M}\left\{\left|\xi_{i}-\xi\right| \geq \varepsilon\right\}=0
$$

for every $\varepsilon>0$.

Remark 5. According to Definition 2.3 and Corollary 3.2 in You [11], we know there exists subsequence $\left\{\xi_{i k}\right\}$ such that $\lim _{k \rightarrow \infty} \mathscr{M}\left\{\left|\xi_{i k}-\xi\right|<\varepsilon\right\}=1$. That is to say, if uncertain sequence $\left\{\xi_{i}\right\}$ converges in measure to $\xi$, we can get $\lim _{k \rightarrow \infty}\left|\xi_{i k}-\xi\right|=0$, a.s.

Definition 6 (see You and Yan [19]). The uncertain sequence $\left\{\xi_{i}\right\}$ is said to be convergent in $p$-distance to $\xi$ if

$$
\lim _{i, j \rightarrow \infty} d_{p}\left(\xi_{i}, \xi_{j}\right)=\lim _{i, j \rightarrow \infty}\left(E\left[\left|\xi_{i}-\xi_{j}\right|^{p}\right]\right)^{1 /(p+1)}=0 .
$$

Theorem 7 (see You and Yan [19]). Let $\xi, \eta$, and $\tau$ be uncertain variables, and let $d_{p}(\cdot, \cdot)$ be the $p$-distance. Then we have the following:
(a) (Nonnegativity) $d_{p}(\xi, \eta) \geq 0$.
(b) (Identification) $d_{p}(\xi, \eta)=0$ if and only if $\xi=\eta$.

(c) $\left(\right.$ Symmetry) $d_{p}(\xi, \eta)=d_{p}(\eta, \xi)$.

(d) (Triangle Inequality) $d_{p}(\xi, \eta) \leq d_{p}(\xi, \tau)+d_{p}(\tau, \eta)$.

Theorem 8 (see You and Yan [20]). Let $\xi$, $\eta$ be uncertain variables defined on metric space $\left(\mathscr{F}, d_{p}\right)$, and let $\left\{\xi_{i}\right\},\left\{\eta_{i}\right\}$ be uncertain variables sequences defined on metric space $\left(\mathscr{F}, d_{p}\right)$. Thus we obtain the following conclusions:

(a) If $\lim _{i \rightarrow \infty} d_{p}\left(\xi_{i}, \xi\right)=0$, then $\lim _{i \rightarrow \infty} d_{p}\left(\left|\xi_{i}\right|,|\xi|\right)=0$.

(b) If $\lim _{i \rightarrow \infty} d_{p}\left(\xi_{i}, \xi\right)=0$ and $\lim _{i \rightarrow \infty} d_{p}\left(\xi_{i}, \eta\right)=0$, then $\xi=\eta$.

(c) If $\lim _{i \rightarrow \infty} d_{p}\left(\xi_{i}, \xi\right)=0$ and $\lim _{i \rightarrow \infty} d_{p}\left(\eta_{i}, \eta\right)=0$, then $\lim _{i \rightarrow \infty} d_{p}\left(a \xi_{i}+b \eta_{i}, a \xi+b \eta\right)=0$, for any real numbers $a, b$.

(d) If $\lim _{i \rightarrow \infty} d_{p}\left(\xi_{i}, \xi\right)=0, \lim _{i \rightarrow \infty} d_{p}\left(\eta_{i}, \eta\right)=0$, and there exists a real number $M>0$ such that $|\xi| \leq M$ and $|\eta| \leq M$, then $\lim _{i \rightarrow \infty} d_{p}\left(\xi_{i} \eta_{i}, \xi \eta\right)=0$.

\section{Some Properties of Convergence}

This section will describe some properties of convergence in measure and convergence in $p$-distance.

Theorem 9. Suppose that uncertain variables sequences $\left\{\xi_{n}\right\}$, $\left\{\eta_{n}\right\}$ converge in measure to $\xi$, $\eta$, respectively. If there exist positive numbers $M_{1}, M, N_{1}$, and $N$ such that $M_{1} \leq\left|\xi_{n}\right| \leq M$ and $N_{1} \leq\left|\eta_{n}\right| \leq N$, for any $n$, then we have the following:

(1) $\left\{\xi_{n}+\eta_{n}\right\}$ converges in measure to $\{\xi+\eta\}$.

(2) $\left\{\xi_{n}-\eta_{n}\right\}$ converges in measure to $\{\xi-\eta\}$.

(3) $\left\{\xi_{n} \eta_{n}\right\}$ converges in measure to $\{\xi \eta\}$.

(4) $\left\{\xi_{n} / \eta_{n}\right\}$ converges in measure to $\{\xi / \eta\}$.

Proof. Since uncertain sequence $\left\{\xi_{i}\right\}$ converges in measure to $\xi$, it follows from Remark 5 that there exists subsequence $\left\{\xi_{i k}\right\}$ such that $\lim _{k \rightarrow \infty}\left|\xi_{i k}-\xi\right|=0$, a.s. Since $M_{1} \leq\left|\xi_{n}\right| \leq M$, we have $M_{1} \leq|\xi| \leq M$. In a similar way, if $N_{1} \leq\left|\eta_{n}\right| \leq N$, then $N_{1} \leq|\eta| \leq N$.

(1) It follows from Axiom 3 that

$$
\begin{aligned}
\mathscr{M} & \left\{\left|\left(\xi_{n}+\eta_{n}\right)-(\xi+\eta)\right| \geq \varepsilon\right\} \\
& =\mathscr{M}\left\{\left|\left(\xi_{n}-\xi\right)+\left(\eta_{n}-\eta\right)\right| \geq \varepsilon\right\} \\
& \leq \mathscr{M}\left\{\left|\xi_{n}-\xi\right| \geq \frac{\varepsilon}{2}\right\}+\mathscr{M}\left\{\left|\eta_{n}-\eta\right| \geq \frac{\varepsilon}{2}\right\} \longrightarrow 0,
\end{aligned}
$$

as $n \rightarrow 0$. Thus uncertain variables sequence $\left\{\xi_{n}+\eta_{n}\right\}$ converges in measure to $\{\xi+\eta\}$.

(2) According to Axiom 3, we obtain

$$
\begin{aligned}
\mathscr{M} & \left\{\left|\left(\xi_{n}-\eta_{n}\right)-(\xi-\eta)\right| \geq \varepsilon\right\} \\
& =\mathscr{M}\left\{\left|\left(\xi_{n}-\xi\right)-\left(\eta_{n}-\eta\right)\right| \geq \varepsilon\right\} \\
& \leq \mathscr{M}\left\{\left|\xi_{n}-\xi\right| \geq \frac{\varepsilon}{2}\right\}+\mathscr{M}\left\{\left|\eta_{n}-\eta\right| \geq \frac{\varepsilon}{2}\right\} \rightarrow 0,
\end{aligned}
$$

as $n \rightarrow 0$. Therefore, uncertain variables sequence $\left\{\xi_{n}-\eta_{n}\right\}$ converges in measure to $\{\xi-\eta\}$. 
(3) By Axiom 3, we have

$$
\begin{aligned}
\mathscr{M} & \left\{\left|\xi_{n} \eta_{n}-\xi \eta\right| \geq \varepsilon\right\}=\mathscr{M}\left\{\left|\xi_{n} \eta_{n}-\xi_{n} \eta+\xi_{n} \eta-\xi \eta\right| \geq \varepsilon\right\} \\
& \leq \mathscr{M}\left\{\left|\xi_{n} \eta_{n}-\xi_{n} \eta\right| \geq \frac{\varepsilon}{2}\right\}+\mathscr{M}\left\{\left|\xi_{n} \eta-\xi \eta\right| \geq \frac{\varepsilon}{2}\right\} \\
& \leq \mathscr{M}\left\{M\left|\eta_{n}-\eta\right| \geq \frac{\varepsilon}{2}\right\}+\mathscr{M}\left\{N\left|\xi_{n}-\xi\right| \geq \frac{\varepsilon}{2}\right\} \\
& =\mathscr{M}\left\{\left|\eta_{n}-\eta\right| \geq \frac{\varepsilon}{2 M}\right\}+\mathscr{M}\left\{\left|\xi_{n}-\xi\right| \geq \frac{\varepsilon}{2 N}\right\} \\
& \longrightarrow 0
\end{aligned}
$$

as $n \rightarrow 0$. Thus uncertain variables sequence $\left\{\xi_{n} \eta_{n}\right\}$ converges in measure to $\{\xi \eta\}$.

(4) It follows from Axiom 3 that

$$
\begin{aligned}
\mathscr{M} & \left\{\left|\frac{\xi_{n}}{\eta_{n}}-\frac{\xi}{\eta}\right| \geq \varepsilon\right\}=\mathscr{M}\left\{\left|\frac{\xi_{n}}{\eta_{n}}-\frac{\xi_{n}}{\eta}+\frac{\xi_{n}}{\eta}-\frac{\xi}{\eta}\right| \geq \varepsilon\right\} \\
& =\mathscr{M}\left\{\left|\frac{\xi_{n}\left(\eta-\eta_{n}\right)}{\eta_{n} \eta}+\frac{\xi_{n}-\xi}{\eta}\right| \geq \varepsilon\right\} \\
& \leq \mathscr{M}\left\{\left|\frac{\xi_{n}\left(\eta_{n}-\eta\right)}{\eta_{n} \eta}\right| \geq \frac{\varepsilon}{2}\right\}+\mathscr{M}\left\{\left|\frac{\xi_{n}-\xi}{\eta}\right| \geq \frac{\varepsilon}{2}\right\} \\
& \leq \mathscr{M}\left\{\frac{M}{N_{1}^{2}}\left|\eta_{n}-\eta\right| \geq \frac{\varepsilon}{2}\right\}+\mathscr{M}\left\{\frac{1}{N_{1}}\left|\xi_{n}-\xi\right| \geq \frac{\varepsilon}{2}\right\} \\
& =\mathscr{M}\left\{\left|\eta_{n}-\eta\right| \geq \frac{N_{1} N_{1} \varepsilon}{2 M}\right\}+\mathscr{M}\left\{\left|\xi_{n}-\xi\right| \geq \frac{N_{1} \varepsilon}{2}\right\} \\
& \longrightarrow 0
\end{aligned}
$$

as $n \rightarrow 0$. Then we get that uncertain variables sequence $\left\{\xi_{n} / \eta_{n}\right\}$ converges in measure to $\{\xi / \eta\}$.

The following theorem is a generalization of Theorem 9 .

Theorem 10. Suppose that uncertain variables sequences $\left\{\xi_{1 n}\right\},\left\{\xi_{2 n}\right\}, \ldots,\left\{\xi_{k n}\right\}$ converge in measure to $\xi_{1}, \xi_{2}, \ldots, \xi_{k}$, respectively. Assume $Q\left(x_{1}, x_{2}, \ldots, x_{k}\right)$ is rational function. If there exist positive numbers $M_{i}^{\prime}, M_{i}$ such that $M_{i}^{\prime} \leq\left|\xi_{i}\right| \leq$ $M_{i}(i=1,2, \ldots, k)$ and $Q\left(\xi_{1}, \xi_{2}, \ldots, \xi_{k}\right) \neq \pm \infty$, then $Q\left(\xi_{1 n}, \xi_{2 n}, \ldots, \xi_{k n}\right)$ converges in measure to $Q\left(\xi_{1}, \xi_{2}, \ldots, \xi_{k}\right)$.

Proof. According to Theorem 9, we have

$$
\begin{aligned}
\mathscr{M}\left\{\left|Q\left(\xi_{1 n}, \xi_{2 n}, \ldots, \xi_{k n}\right)-Q\left(\xi_{1}, \xi_{2}, \ldots, \xi_{k}\right)\right| \geq \varepsilon\right\} \\
\leq \mathscr{M}\left\{\left|\xi_{1 n}-\xi_{1}\right| \geq \frac{\varepsilon}{k}\right\}+\mathscr{M}\left\{\left|\xi_{2 n}-\xi_{2}\right| \geq \frac{\varepsilon}{k}\right\}+\cdots \\
\quad+\mathscr{M}\left\{\left|\xi_{k n}-\xi_{k}\right| \geq \frac{\varepsilon}{k}\right\} \longrightarrow 0,
\end{aligned}
$$

as $n \rightarrow \infty$. The proof is completed.

Theorem 11. Assume uncertain variables sequence $\left\{\xi_{n}\right\}$ converges in measure to $\xi$. If $f(x)$ is a continuous function, then $f\left(\xi_{n}\right)$ converges in measure to $f(\xi)$.
Proof. (1) If $f(x)=\sum_{i=1}^{k} a_{i} x^{i}$ is $k$-order polynomial function, by Theorem 10, we can get that $f\left(\xi_{n}\right)$ converges in measure to $f(\xi)$.

(2) Since $\left\{\xi_{n}\right\}$ converges in measure to $\xi$, there exists a positive number $M_{0}$ such that $\mathscr{M}\left\{|\xi|>M_{0}\right\}<\delta$, for any $0<\delta<1$. Since $\left\{\xi_{n}\right\}$ converges in measure to $\xi$, there exists positive number $N_{1}$ such that $\mathscr{M}\left\{\left|\xi-\xi_{n}\right|>1\right\}<\delta$, for any $n \geq N_{1}$. Thus

$$
\begin{aligned}
\mathscr{M}\left\{\left|\xi_{n}\right| \geq M_{0}+1\right\} \leq & \mathscr{M}\left\{|\xi|>M_{0}\right\} \\
& +\mathscr{M}\left\{\left|\xi-\xi_{n}\right|>1\right\}<2 \delta .
\end{aligned}
$$

Since $f(x)$ is a continuous function, there exists a $k$-order polynomial function $g_{k}(x)$ such that

$$
\left|f(x)-g_{k}(x)\right|<\frac{\varepsilon}{3} .
$$

It follows from (1) that $g_{k}\left(\xi_{n}\right)$ converges in measure to $g_{k}(\xi)$. That is to say, there exists a positive integer $N_{2}$ such that

$$
\mathscr{M}\left\{\left|g_{k}\left(\xi_{n}\right)-g_{k}(\xi)\right| \geq \frac{\varepsilon}{3}\right\}<\delta
$$

for any $n \geq N_{2}$.

Then

$$
\begin{aligned}
\mathscr{M} & \left\{\left|f(\xi)-f\left(\xi_{n}\right)\right| \geq \varepsilon\right\} \\
\leq & \mathscr{M}\left\{\left\{\left|f(\xi)-f\left(\xi_{n}\right)\right| \geq \varepsilon\right\} \cap\{A \cup B\}\right\} \\
& +\mathscr{M}\left\{\left\{\left|f(\xi)-f\left(\xi_{n}\right)\right| \geq \varepsilon\right\} \cap\{\overline{A \cup B}\}\right\} \\
= & I_{1}+I_{2},
\end{aligned}
$$

where

$$
\begin{aligned}
\{A \cup B\} & =\left\{|\xi|>M_{0}\right\} \cup\left\{\left|\xi_{n}\right|>M_{0}+1\right\}, \\
\{\overline{A \cup B}\} & =\{\bar{A} \cap \bar{B}\}=\left\{|\xi| \leq M_{0}\right\} \cap\left\{\left|\xi_{n}\right| \leq M_{0}+1\right\}, \\
I_{1} & =\mathscr{M}\left\{\left\{\left|f(\xi)-f\left(\xi_{n}\right)\right| \geq \varepsilon\right\} \cap\{A \cup B\}\right\}, \\
I_{2} & =\mathscr{M}\left\{\left\{\left|f(\xi)-f\left(\xi_{n}\right)\right| \geq \varepsilon\right\} \cap\{\overline{A \cup B}\}\right\} .
\end{aligned}
$$

Furthermore, we have

$$
\begin{aligned}
I_{1} & =\mathscr{M}\left\{\left\{\left|f(\xi)-f\left(\xi_{n}\right)\right| \geq \varepsilon\right\}\right. \\
& \left.\cap\left\{\left\{|\xi|>M_{0}\right\} \cup\left\{\left|\xi_{n}\right|>M_{0}+1\right\}\right\}\right\} \\
& \leq \mathscr{M}\left\{\left\{|\xi|>M_{0}\right\} \cup\left\{\left|\xi_{n}\right|>M_{0}+1\right\}\right\} \leq \mathscr{M}\{|\xi| \\
& \left.>M_{0}\right\}+\mathscr{M}\left\{\left|\xi_{n}\right|>M_{0}+1\right\}<3 \delta .
\end{aligned}
$$

Since

$$
\begin{aligned}
& \left\{\left|f(\xi)-f\left(\xi_{n}\right)\right| \geq \varepsilon\right\}=\left\{\mid f(\xi)-g_{k}(\xi)+g_{k}(\xi)\right. \\
& \left.\quad-g_{k}\left(\xi_{n}\right)+g_{k}\left(\xi_{n}\right)-f\left(\xi_{n}\right) \mid \geq \varepsilon\right\},
\end{aligned}
$$


we obtain $\left|f(\xi)-g_{k}(\xi)\right| \geq \varepsilon / 3$ or $\left|g_{k}(\xi)-g_{k}\left(\xi_{n}\right)\right| \geq \varepsilon / 3$ or $\left|g_{k}\left(\xi_{n}\right)-f\left(\xi_{n}\right)\right| \geq \varepsilon / 3$. That is,

$$
\begin{aligned}
& \left\{\left|f(\xi)-f\left(\xi_{n}\right)\right| \geq \varepsilon\right\} \subset\left\{\left\{\left|f(\xi)-g_{k}(\xi)\right| \geq \frac{\varepsilon}{3}\right\}\right. \\
& \cup\left\{\left|g_{k}(\xi)-g_{k}\left(\xi_{n}\right)\right| \geq \frac{\varepsilon}{3}\right\} \\
& \left.\cup\left\{\left|g_{k}\left(\xi_{n}\right)-f\left(\xi_{n}\right)\right| \geq \frac{\varepsilon}{3}\right\}\right\},
\end{aligned}
$$

By the reason that

$$
\begin{aligned}
& \mathscr{M}\left\{\left\{\left|f(\xi)-g_{k}(\xi)\right| \geq \frac{\varepsilon}{3}\right\} \cap\{\overline{A \cup B}\}\right\} \\
& =\mathscr{M}\left\{\left\{\left|f(\xi)-g_{k}(\xi)\right| \geq \frac{\varepsilon}{3}\right\} \cap\left\{|\xi| \leq M_{0}\right\}\right. \\
& \left.\cap\left\{\left|\xi_{n}\right| \leq M_{0}+1\right\}\right\}=0, \\
& \mathscr{M}\left\{\left\{\left|g_{k}\left(\xi_{n}\right)-f\left(\xi_{n}\right)\right| \geq \frac{\varepsilon}{3}\right\} \cap\{\overline{A \cup B}\}\right\} \\
& =\mathscr{M}\left\{\left\{\left|g_{k}\left(\xi_{n}\right)-f\left(\xi_{n}\right)\right| \geq \frac{\varepsilon}{3}\right\} \cap\left\{|\xi| \leq M_{0}\right\}\right. \\
& \left.\cap\left\{\left|\xi_{n}\right| \leq M_{0}+1\right\}\right\}=0 .
\end{aligned}
$$

we have

$$
\begin{aligned}
I_{2} & \leq \mathscr{M}\left\{\left\{\left|g_{k}(\xi)-g_{k}\left(\xi_{n}\right)\right| \geq \frac{\varepsilon}{3}\right\} \cap\left\{|\xi| \leq M_{0}\right\}\right. \\
& \left.\cap\left\{\left|\xi_{n}\right| \leq M_{0}+1\right\}\right\} \leq \mathscr{M}\left\{\left|g_{k}(\xi)-g_{k}\left(\xi_{n}\right)\right| \geq \frac{\varepsilon}{3}\right\} \\
& <\delta .
\end{aligned}
$$

Therefore,

$$
\mathscr{M}\left\{\left|f(\xi)-g_{k}(\xi)\right| \geq \varepsilon\right\} \leq I_{1}+I_{2}<4 \delta .
$$

By the arbitrariness of $\varepsilon, \delta$, we can get that $f\left(\xi_{n}\right)$ converges in measure to $f(\xi)$.

Based on Theorems 10 and 11, the following theorem was acquired.

Theorem 12. If uncertain variables sequences $\left\{\xi_{i n}\right\}, i=$ $1,2, \ldots, k$, converge in measure to $\xi_{i}$, respectively, and $Q\left(g_{1}\left(\xi_{1}\right), g_{2}\left(\xi_{2}\right), \ldots, g_{k}\left(\xi_{k}\right)\right) \neq \pm \infty$, for any rational function $Q\left(x_{1}, x_{2}, \ldots, x_{k}\right)$ and continuous function $g_{i}(x), i=$ $1,2, \ldots, k$, then $Q\left(g_{1}\left(\xi_{1 n}\right), g_{2}\left(\xi_{2 n}\right), \ldots, g_{k}\left(\xi_{k n}\right)\right)$ converges in measure to $Q\left(g_{1}\left(\xi_{1}\right), g_{2}\left(\xi_{2}\right), \ldots, g_{k}\left(\xi_{k}\right)\right)$.

Proof. The theorem can be proved easily by using Theorems 10 and 11.

Theorem 13. If uncertain variables sequence $\left\{\xi_{n}\right\}$ converges in measure to $\xi, \eta$, respectively, then $\mathscr{M}\{\xi=\eta\}=1$.
Proof. According to Axiom 3, we obtain

$$
\begin{aligned}
0 & \leq \mathscr{M}\{|\xi-\eta| \geq \varepsilon\}=\mathscr{M}\left\{\left|\xi-\xi_{n}+\xi_{n}-\eta\right| \geq \varepsilon\right\} \\
& \leq \mathscr{M}\left\{\left|\xi-\xi_{n}\right| \geq \frac{\varepsilon}{2}\right\}+\mathscr{M}\left\{\left|\eta-\xi_{n}\right| \geq \frac{\varepsilon}{2}\right\} \longrightarrow 0
\end{aligned}
$$

as $n \rightarrow \infty$. Thus the theorem is proved.

Next, the results of convergence in measure will be extended to the case of convergence in $p$-distance.

Theorem 14. Suppose that uncertain variables sequences $\left\{\xi_{n}\right\}$, $\left\{\eta_{n}\right\}$ converge in p-distance to $\xi$, $\eta$, respectively. If there exist positive numbers $M_{1}, M, N_{1}$, and $N$ such that $M_{1} \leq\left|\xi_{n}\right| \leq M$ and $N_{1} \leq \eta_{n} \leq N$, for any $n$, then

(1) $\lim _{i \rightarrow \infty} d_{p}\left(\xi_{i}-\eta_{i}, \xi-\eta\right)=0$,

(2) $\lim _{i \rightarrow \infty} d_{p}\left(\xi_{i} / \eta_{i}, \xi / \eta\right)=0$.

Proof. (1) It follows from Triangle Inequality that

$$
\begin{aligned}
0 \leq & \lim _{i \rightarrow \infty} d_{p}\left(\xi_{i}-\eta_{i}, \xi-\eta\right) \\
\leq & \lim _{i \rightarrow \infty} d_{p}\left(\xi_{i}-\eta_{i}, \xi_{i}-\eta\right)+\lim _{i \rightarrow \infty} d_{p}\left(\xi_{i}-\eta, \xi-\eta\right) \\
= & \lim _{i \rightarrow \infty}\left(E\left[\left|\left(\xi_{i}-\eta_{i}\right)-\left(\xi_{i}-\eta\right)\right|^{p}\right]\right)^{1 /(p+1)} \\
& +\lim _{i \rightarrow \infty}\left(E\left[\left|\left(\xi_{i}-\eta\right)-(\xi-\eta)\right|^{p}\right]\right)^{1 /(p+1)} \\
= & \lim _{i \rightarrow \infty}\left(E\left[\left|\eta_{i}-\eta\right|^{p}\right]\right)^{1 /(p+1)} \\
& +\lim _{i \rightarrow \infty}\left(E\left[\left|\xi_{i}-\xi\right|^{p}\right]\right)^{1 /(p+1)} \\
= & \lim _{i \rightarrow \infty} d_{p}\left(\eta_{i}, \eta\right)+\lim _{i \rightarrow \infty} d_{p}\left(\xi_{i}, \xi\right)=0 .
\end{aligned}
$$

Thus, $\lim _{i \rightarrow \infty} d_{p}\left(\xi_{i}-\eta_{i}, \xi-\eta\right)=0$.

(2) Suppose that $\Gamma^{+}$is the set of points which have positive uncertain measure. For any $\gamma \in \Gamma^{+}$,

$$
\begin{aligned}
\lim _{i \rightarrow \infty}\left|\xi_{i}(\gamma)-\xi(\gamma)\right| & \leq \lim _{i \rightarrow \infty} \frac{E\left[\left|\xi_{i}-\xi\right|^{p}\right]}{\mathscr{M}\{\gamma\}} \\
& =\lim _{i \rightarrow \infty} \frac{d_{p}^{p+1}\left(\xi_{i}, \xi\right)}{\mathscr{M}\{\gamma\}}=0 .
\end{aligned}
$$

Since $0<M_{1} \leq\left|\xi_{n}\right| \leq M$, we have $M_{1} \leq|\xi| \leq M$. In a similar way, if $N_{1} \leq\left|\eta_{n}\right| \leq N$, then $N_{1} \leq|\eta| \leq N$.

By Triangle Inequality, we can get

$$
\begin{aligned}
0 & \leq \lim _{i \rightarrow \infty} d_{p}\left(\frac{\xi_{i}}{\eta_{i}}, \frac{\xi}{\eta}\right) \\
& \leq \lim _{i \rightarrow \infty} d_{p}\left(\frac{\xi_{i}}{\eta_{i}}, \frac{\xi_{i}}{\eta}\right)+\lim _{i \rightarrow \infty} d_{p}\left(\frac{\xi_{i}}{\eta}, \frac{\xi}{\eta}\right)
\end{aligned}
$$




$$
\begin{aligned}
= & \lim _{i \rightarrow \infty}\left(E\left[\left|\frac{\xi_{i}}{\eta_{i}}-\frac{\xi_{i}}{\eta}\right|^{p}\right]\right)^{1 /(p+1)} \\
& +\lim _{i \rightarrow \infty}\left(E\left[\left|\frac{\xi_{i}}{\eta}-\frac{\xi}{\eta}\right|^{p}\right]\right)^{1 /(p+1)} \\
= & \lim _{i \rightarrow \infty}\left(E\left[\left|\frac{\xi_{i}\left(\eta-\eta_{i}\right)}{\eta_{i} \eta}\right|^{p}\right]\right)^{1 /(p+1)} \\
& +\lim _{i \rightarrow \infty}\left(E\left[\left|\frac{\xi_{i}-\xi}{\eta}\right|^{p}\right]\right)^{1 /(p+1)} \\
\leq & \left(\frac{M}{N_{1}^{2}}\right)^{p /(p+1)} \lim _{i \rightarrow \infty} d_{p}\left(\eta_{i}, \eta\right) \\
& +\left(\frac{1}{N_{1}}\right)^{p /(p+1)} \lim _{i \rightarrow \infty} d_{p}\left(\xi_{i}, \xi\right)=0 .
\end{aligned}
$$

Thus $\lim _{i \rightarrow \infty} d_{p}\left(\xi_{i} / \eta_{i}, \xi / \eta\right)=0$ is verified.

Remark 15. According to Theorems 8 and 14, we know the arithmetic operation of uncertain sequences, which are convergent in $p$-distance, are still convergent in $p$-distance.

Theorem 16. Suppose that uncertain variables sequences $\left\{\xi_{1 n}\right\},\left\{\xi_{2 n}\right\}, \ldots,\left\{\xi_{k n}\right\}$ converge in $p$-distance to $\xi_{1}, \xi_{2}, \ldots, \xi_{k}$, respectively. Assume $Q\left(x_{1}, x_{2}, \ldots, x_{k}\right)$ is rational function. If there exist positive numbers $M_{i}^{\prime}, M_{i}$ such that $M_{i}^{\prime} \leq\left|\xi_{i}\right| \leq$ $M_{i}(i=1,2, \ldots, k)$ and $Q\left(\xi_{1}, \xi_{2}, \ldots, \xi_{k}\right) \neq \pm \infty$, then $Q\left(\xi_{1 n}, \xi_{2 n}, \ldots, \xi_{k n}\right)$ converges in $p$-distance to $Q\left(\xi_{1}, \xi_{2}, \ldots, \xi_{k}\right)$.

Proof. It follows from Theorems 14 and 8 that

$$
\begin{aligned}
\lim _{i \rightarrow \infty} d_{p}\left(Q\left(\xi_{1 n}, \xi_{2 n}, \ldots, \xi_{k n}\right), Q\left(\xi_{1}, \xi_{2}, \ldots, \xi_{k}\right)\right) \\
\leq \lim _{i \rightarrow \infty} d_{p}\left(\xi_{1 n}, \xi_{1}\right)+\lim _{i \rightarrow \infty} d_{p}\left(\xi_{2 n}, \xi_{2}\right)+\cdots \\
\quad+\lim _{i \rightarrow \infty} d_{p}\left(\xi_{k n}, \xi_{k}\right)=0 .
\end{aligned}
$$

Therefore, $Q\left(\xi_{1 n}, \xi_{2 n}, \ldots, \xi_{k n}\right)$ converges in $p$-distance to $\mathrm{Q}\left(\xi_{1}, \xi_{2}, \ldots, \xi_{k}\right)$.

Theorem 17. If uncertain variables sequence $\left\{\xi_{n}\right\}$ converges in $p$-distance to uncertain variable $\xi$, then $f\left(\xi_{n}\right)$ converges in $p$ distance to $f(\xi)$, for any continuous function $f(x)$.

Proof. (1) If $f(x)=\sum_{i=1}^{k} a_{i} x^{i}$ is $k$-order polynomial function, according to Theorem 10 , we know that $f\left(\xi_{n}\right)$ converges in $p$-distance to $f(\xi)$.

(2) If $f(x)$ is arbitrary continuous function, there exists a $k$-order polynomial function $g_{k}(x)$ such that

$$
\left|f(x)-g_{k}(x)\right|<\left(\frac{\varepsilon}{3}\right)^{(p+1) / p}
$$

for any $\varepsilon>0$. That is to say,

$$
\begin{aligned}
d_{p}\left(f(x), g_{k}(x)\right) & =\left(E\left[\left|f(x)-g_{k}(x)\right|^{p}\right]\right)^{1 /(p+1)} \\
& <\frac{\varepsilon}{3}
\end{aligned}
$$

From (1), we obtain that $g_{k}\left(\xi_{n}\right)$ converges in $p$-distance to $g_{k}(\xi)$, for any positive number $k$. That is to say, there exists positive integer $N$, such that

$$
d_{p}\left(g_{k}\left(\xi_{n}\right), g_{k}(\xi)\right)<\frac{\varepsilon}{3},
$$

for any $n>N$.

Thus, we get

$$
\begin{aligned}
d_{p}\left(f\left(\xi_{n}\right), f(\xi)\right) \leq & d_{p}\left(f\left(\xi_{n}\right), g_{n}\left(\xi_{n}\right)\right) \\
& +d_{p}\left(g_{n}\left(\xi_{n}\right), f(\xi)\right) \\
\leq & d_{p}\left(f\left(\xi_{n}\right), g_{n}\left(\xi_{n}\right)\right) \\
& +d_{p}\left(g_{n}\left(\xi_{n}\right), g_{n}(\xi)\right) \\
& +d_{p}\left(g_{n}(\xi), f(\xi)\right) \\
< & \frac{\varepsilon}{3}+\frac{\varepsilon}{3}+\frac{\varepsilon}{3}=\varepsilon,
\end{aligned}
$$

for any $n>N$.

Therefore, $f\left(\xi_{n}\right)$ converges in $p$-distance to $\xi$.

\section{Conclusions}

The main point of this paper is to discuss properties of convergence for uncertain sequence. To begin with, some properties of convergence in measure were investigated in this paper; for example, the arithmetic operation of uncertain sequence, which are convergent in measure, are still convergent in measure. If an uncertain sequence is convergent in measure, then the sequence formed by the continuous function of the uncertain sequence is convergent in measure too and so forth. Then the results of convergence in measure were extended to the case of convergence in $p$-distance.

Next, we will investigate the corresponding convergence properties of uncertain sequence in the sense of convergence almost surely and convergence in distribution and other convergence concepts. Furthermore, we will apply these convergence properties into the real problems in engineering and mathematical finance.

\section{Conflicts of Interest}

The authors declare that they have no conflicts of interest with regard to the publication of this manuscript.

\section{Acknowledgments}

This work was supported by Natural Science Foundation of China, Grant no. 61374184. 


\section{References}

[1] B. Liu, Uncertainty Theory, Springer-Verlag, Berlin, 2nd edition, 2007.

[2] B. Liu, "Uncertain risk analysis and uncertain reliablity analysis," Journal of Uncertain Systems, vol. 4, no. 3, pp. 163-170, 2010.

[3] Y.H. Liu and M. H. Ha, "Expected value of function of uncertain variables," Journal of Uncertain Systems, vol. 4, no. 3, pp. 181-186, 2010.

[4] B. Liu, Theory and Practice of Uncertain Programming, Springer, Berlin, 2nd edition, 2009.

[5] X. Li and B. Liu, "Hybrid logic and uncertain logic," Journal of Uncertain Systems, vol. 3, no. 2, pp. 83-94, 2009.

[6] B. Liu, "Fuzzy process, hybrid process and uncertain process," Journal of Uncertain Systems, vol. 2, no. 1, pp. 3-16, 2008.

[7] B. Liu, "Uncertainty distribution and independence of uncertain processes," Fuzzy Optimization and Decision Making, vol. 13, no. 3, pp. 259-271, 2014.

[8] X. Chen and B. Liu, "Existence and uniqueness theorem for uncertain differential equations," Fuzzy Optimization and Decision Making, vol. 9, no. 1, pp. 69-81, 2010.

[9] B. Liu, "Uncertain set theory and uncertain inference rule with application to uncertain control," Journal of Uncertain Systems, vol. 4, no. 2, pp. 83-98, 2010.

[10] X. Gao, Y. Gao, and D. A. Ralescu, "On Liu's inference rule for uncertain systems," International Journal of Uncertainty, Fuzziness and Knowledge-Based Systems, vol. 18, no. 1, pp. 1-11, 2010.

[11] C. You, "On the convergence of uncertain sequences," Mathematical and Computer Modelling, vol. 49, no. 3-4, pp. 482-487, 2009.

[12] Y. Xia, Convergence of uncertain sequences [Dissertation, thesis], Suzhou University of Science and Technology, 2011.

[13] H. Wang and Z. Wang, "The dual limits of an uncertain variable sequence," Fuzzy Systems and Mathematics, vol. 27, no. 1, pp. 137-141, 2013.

[14] X. Gao, "Some properties of continuous uncertain measure," International Journal of Uncertainty, Fuzziness and KnowledgeBased Systems, vol. 17, no. 3, pp. 419-426, 2009.

[15] Y. Guo, J. Q. Zhu, and Y. Yuan, "Research of convergence in measure for uncertain sequences," Journal of Zhengzhou University. Natural Science Edition, vol. 46, no. 3, pp. 28-31, 2014.

[16] Y. Yuan, R-order convergence and dual convergence of uncertain sequences [Dissertation, thesis], Suzhou University of Science and Technology, 2014.

[17] Y. Yuan, J. Zhu, and Y. Guo, "The dual convergence of uncertain variables," Journal of Anyang Teachers College (Natural Science Edition), vol. 2, no. 3, pp. 4-6, 2014.

[18] X. Chen, Y. Ning, and X. Wang, "Convergence of complex uncertain sequences," Journal of Intelligent \& Fuzzy Systems: Applications in Engineering and Technology, vol. 30, no. 6, pp. 3357-3366, 2016.

[19] C. You and L. Yan, “The p-distance of uncertain variables," Journal of Intelligent \& Fuzzy Systems: Applications in Engineering and Technology, vol. 32, no. 1, pp. 999-1006, 2017.

[20] C. You and L. Yan, "Relationships among convergence concepts of uncertain sequences," Computer Modelling and New Technologies, vol. 20, no. 3, pp. 12-16, 2016. 


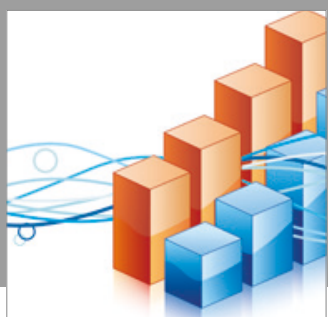

Advances in

Operations Research

vatersals

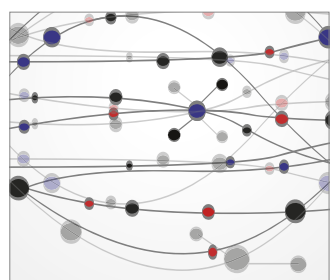

\section{The Scientific} World Journal
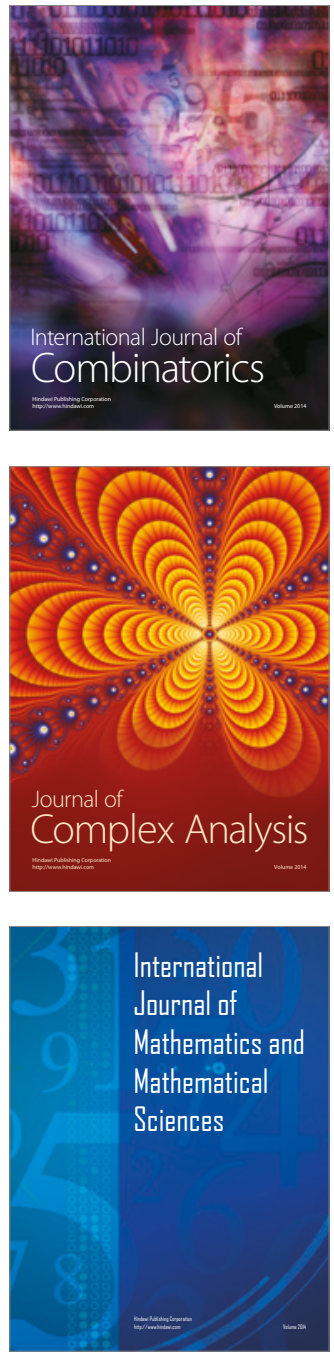
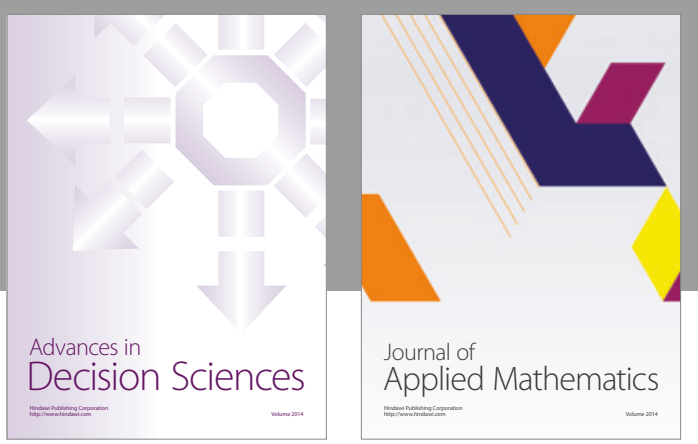

Algebra

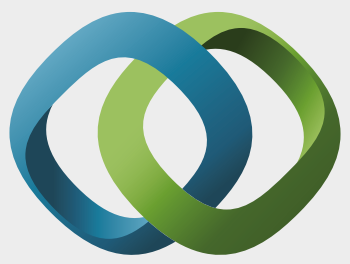

\section{Hindawi}

Submit your manuscripts at

https://www.hindawi.com
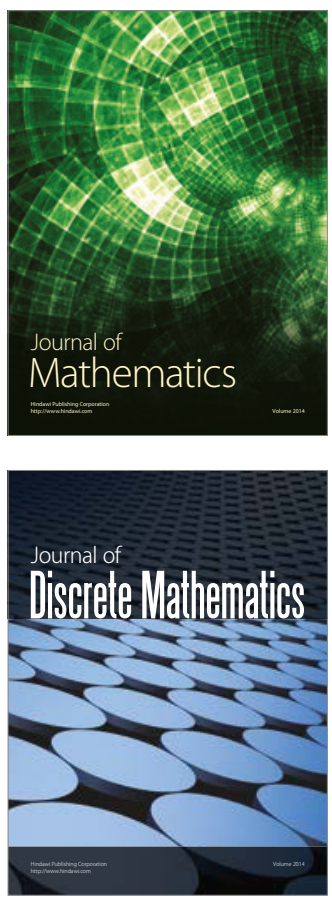

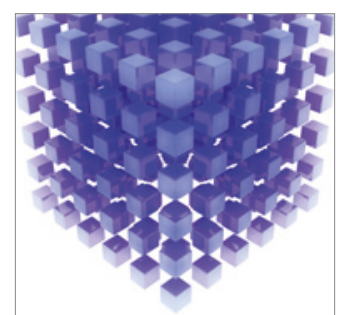

Mathematical Problems in Engineering
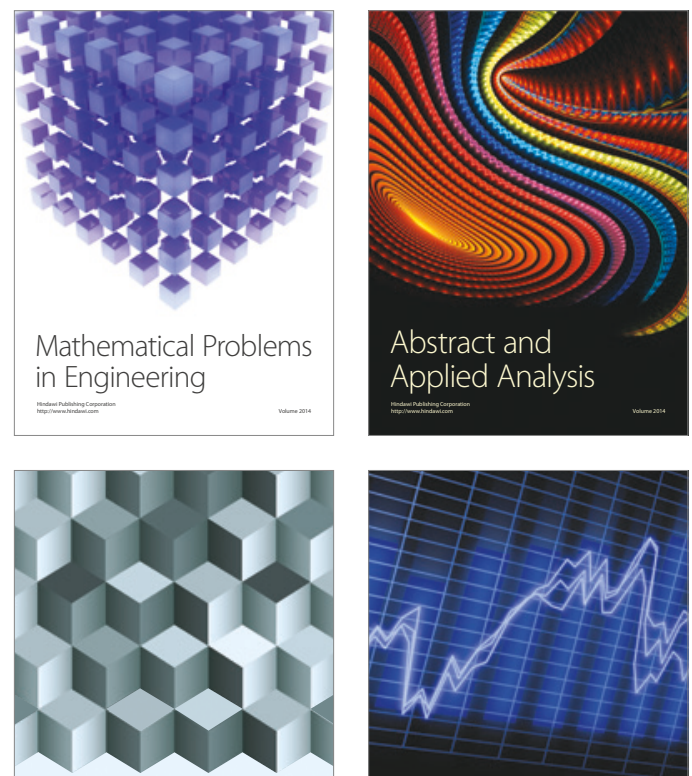

Journal of

Function Spaces

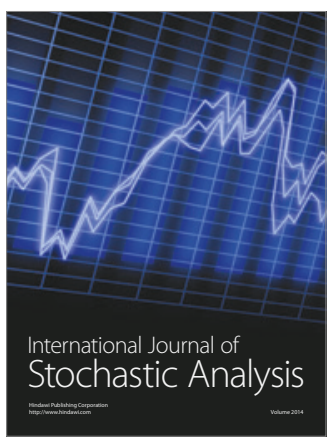

Probability and Statistics
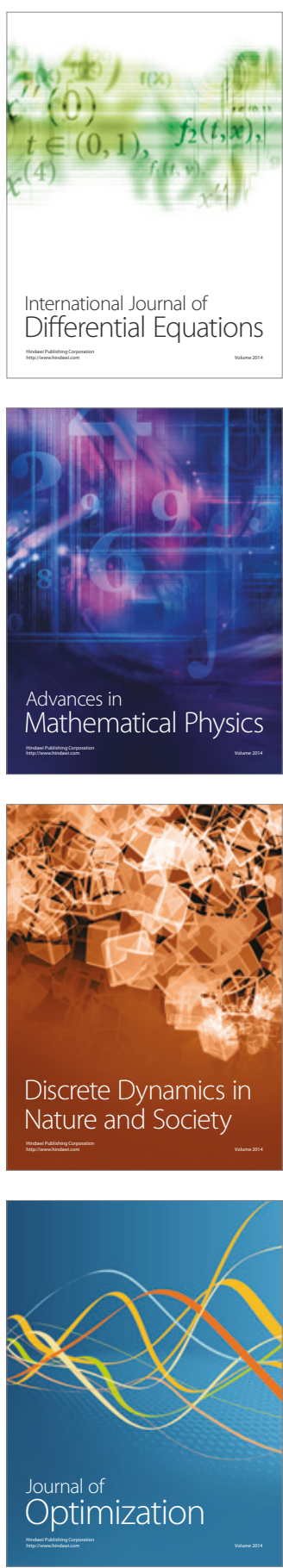